\title{
Attendance of Patients with ophthalmological problems about Eye Protection from UV/blue Light Radiation from Sun light and Electronic Devices in Sulaimani City
}

\author{
Salah Qadir Mahmood \\ Radiation Department \\ Sulaimani Technical Institute \\ Sulaimani Polytechnic University \\ Sulaimani, Iraq \\ salahdeanoffice@hotmail.com \\ Abdulrahman M. Ibrahem \\ Piramagrun Hospital \\ Sulaimani General Directorate of Health \\ Ministry of Health \\ Sulaimani, Iraq \\ abdlrahman.ibrahem@spu.edu.iq
}

\author{
Muhammad Babakir-Mina \\ Medical Laboratory Analysis Department \\ Technical College of Health \\ Sulaimani Polytechnic University \\ Sulaimani, Iraq \\ m.babakir@yahoo.com
}
Bakhtyar Kamal Talabany
Anesthesia Department
Sulaimani Technical Institute
Sulaimani Polytechnic University Sulaimani, Iraq
bakhtyar.talabany@spu.edu.iq

\author{
Salar Ibrahim Ali \\ Community Health Department \\ Technical College of Health \\ Sulaimani Polytechnic University \\ Sulaimani, Iraq \\ salaribrahimali@yahoo.it
}

\begin{abstract}
Ultraviolet (UV) and blue light are affecting eyes during the daily time and may cause many eye's problems and diseases. Therefore, this study aimed to explore the awareness of patients with ophthalmological problems on UV/blue light protection measures and to evaluate their knowledge and protection measures about radiation that emits from sun light and other electronic devices. It comprised a cross-sectional survey on a representative target population with eye diseases who visited the Shahid Aso Eye Hospital at Sulaimani city (sample size $=500$ ). A special designed cross-sectional questionnaire was performed on a random sampling for data collection from $9^{\text {th }}$ September 2017 to $1^{\text {st }}$ January 2018. The statistical analysis was performed using SPSS version 22.0. There was an even distribution between male and female participants across the groups. The highest percentage of patients with light sensitivity was (79.6\%) and the lowest percentage with eye injury was (7.8\%). Most of participants had knowledge about the effects of UV/blue light on eye diseases by (52\%), cataract (57.8\%), glaucoma and red eye with percentage of $(45.8 \%)$ and (43.4\%) respectively. There was a significant difference for the males who were more game-player and using electronic devices like smartphone/tablet /iPad than females. There was a significant difference between male and female participants about the knowledge on harmful effects of radiation from electronic devices. Furthermore, only the patients with sun light sensitivity and cataract had a significant knowledge about harmful effects of radiation from electronic devices on human eyes. Finally, most of the patients with dry eyes, eye/lid infection and glaucoma and cataract had significantly more practices for taking visual breaks when using electronic devices. This study concluded that the patients with ophthalmological problems show a low level of knowledge and protection measurements on the harmful effects of $U V / b l u e$ light on human eyes and radiation protection. Additionally, this study has discovered that sun protection measures are commonly inadequate among the participants and on a regular basis only a small portion of participants were used the sun protection requirements.
\end{abstract}

Keywords: Attendance, Eye disease, UV/Blue light radiation, Eye protection, Electronic devices,

\section{INTRODUCTION}

Electromagnetic radiation (EMR) is the flow of energy at the universal speed of light through free space or through a material medium in the form of the electric and magnetic fields that form electromagnetic waves such as radio waves, visible light, and gamma rays [1]. Blue light is part of the visible light spectrum with short wavelengths which are emitting a higher energy can be divided into two bands: blue-violet light (415-455 nm) and blue-turquoise light (465-495 nm) [2,3]. Blue light has a very short wavelength, and so produces a higher amount of energy. Studies suggest that, over time, exposure to the high energy visible (HEV) light could cause serious long-term damage to the eyes [4]. UV light is electromagnetic radiation with a wavelength shorter than that of visible light, but longer than X-rays. It is so named because the spectrum consists of electromagnetic waves with frequencies higher than those that human eye identifies as the colour violet. Although ultraviolet is invisible to the human eye, most people are aware of the effects of UV through the painful condition of sunburn, but the UV spectrum has many other effects, both beneficial and damaging to human health [5]. The eyes are constantly exposed to solar UV effects, and much evidence attributes pathogenesis of the ocular tissues to such radiation. When UV reaches the eye, the proportion absorbed by different structures depends on the wavelength [6]. Although light is essential to human vision, light exposure can also cause pathological changes in ocular tissues through absorption of photon energy. When absorbed photon energy can be dissipated short wavelength visible light, particularly violet and 
indigo, reaches the retina in substantially greater doses than does ultraviolet (UV) radiation. Indeed, the conditions associated with UV exposure are generally confined to the anterior segment of the eye, due to nearly complete absorption of UV by the crystalline lens [6]. Blue light damage occurs when a photosensitizer of eye absorbs photon energy of a specific wavelength, setting in motion a series of intracellular chemical reactions [6]. Almost all visible blue light passes through the cornea and lens and reaches the retina. This light may affect vision and could prematurely age the eyes. Early research shows that too much exposure to blue light from computer screens and digital devices can decrease contrast leading to digital eyestrain. Some studies suggest that continued exposure to blue light over time could lead to damaged retinal cells. This can cause vision problems like age-related macular degeneration [7]. Therefore, this study aimed to explore the awareness of patients with eye diseases on sun/blue light protection measures and to evaluate their knowledge, attitude and practices about the electromagnetic radiation that emits from the sun light and other electronic devices such as computers, laptops, smartphones, tablets and iPads.

\section{METHODS AND MATERIALS}

Study design and setting: This research was practiced in the Shaheed Aso Eye Hospital at Sulaimani City. It is based on the cross-sectional study. This study was conducted in this hospital because it is the main public eye hospital in the city, which provides services in a wide range of specialties for person in difference age groups who have been referred from the health centres and hospitals, private clinics across the city or from outside of the city. In fact, the majority of ophthalmic therapies of the city occur in this hospital. In addition to that, there are several private eye hospitals providing the services to eye patients.

Target population and sample size: The target population was ophthalmic patients from Sulaimani city at different age and the study population includes all patients who attended the Shaheed Aso Eye Hospital during the period of data collection from ninth September 2017 to first January 2018. A total of 500 eye patients were enrolled in the study.

Case definition and recruitment: A case was defined as any patient consulting at Aso hospital in the different age and residence of Sulaimani governorate and the cases were already diagnosed by the ophthalmologists and the hospital's specialists. All the children less than 10 years old excluded from this study. The cases were recruited four days a week in the morning in the Shaheed Aso Eye Hospital. According to the case definition, the cases were recruited when they reported to the Shaheed Aso Eye Hospital either for initial diagnosis or follow-up processes. Routinely, all patients suspected of having eye disease are referred or admitted to the Shaheed Aso Eye Hospital for diagnosis and management or followup process.
Data Collection and Processing: Data on exposure to the socio-demographic characteristics and the risk factors for human eyes were obtained from the sources. Face to face interview was carried out through a questionnaire and verbal consent was obtained from eye patients. Also, explanation of the study was stated for the patients. Collecting and checking the questionnaire as performed and each day the questionnaire was sorted according to the unique number. For the obtaining, Firstly, the questionnaires were pre-tested, well formatted, pre-coded and questions asked in the native language. Secondly, the interviews were done solely by the researcher himself. Thirdly, the questionnaires were checked daily and completeness was made when is possible. Finally, all data were entered; the data were analysed by Statistical Package for the Social Sciences (SPSS) version 22.0.

\section{RESULTS}

\section{Socio-demographic characteristics}

A total of 500 participants were interviewed. The age of participants ranged from 10 to 87 years old. The mean age (mean \pm SD) for the respondents was (43.54 \pm 21.55 ) with range 77 years old. There was an even distribution of males and females across the groups, of which the majority of the groups were male $59 \%$ (295/500) compared with female $41 \%$ (205/500). Out of 500 respondents $22 \%(110 / 500)$ were in the age group < 20 years old, while others $22.8 \%(114 / 500)$ were in the age group of 20 -39 years old, $25 \%(125 / 500)$ were in the age group of 40 - 59 years old, and $27.4 \%$ (137/500) were in the age group of 60 - 79 years old and $2.8 \%$ $(14 / 500)$ were in the age group of $\geq 80$ (Table 1$)$. Majority of the respondents (61\%) were illiterate or having primary education, $25.6 \%$ were high school educated and $13.4 \%$ were university graduates. Out of 500 respondents, $65.2 \%$ (326) respondents were married, $67.2 \%$ of the respondents were from urban area and rest of the respondents was from semi-urban and rural area. On the other hand, the occupation of participants was unemployed 44.2\% (221/500), government employee $13.6 \%$ (68/500), student $22.6 \%$ $(113 / 500)$ and self-employee was 16.6\%(98/500) (Table 1).

Table 1: Socio-demographic characteristics of participants. 


\section{Attendance of participants on some ophthalmological} problems and scientific terms

The results revealed that the higher percentage of participants' knowledge recorded with blue and UV light and Cataract (52\%) and (57.8\%) respectively. Followed by Glaucoma and red eye by (45.8\%) and (43.4\%) respectively. However, participants' knowledge about trichiasis, diabetic retinopathy, macular degeneration, cross red and pterygium showed very low level.

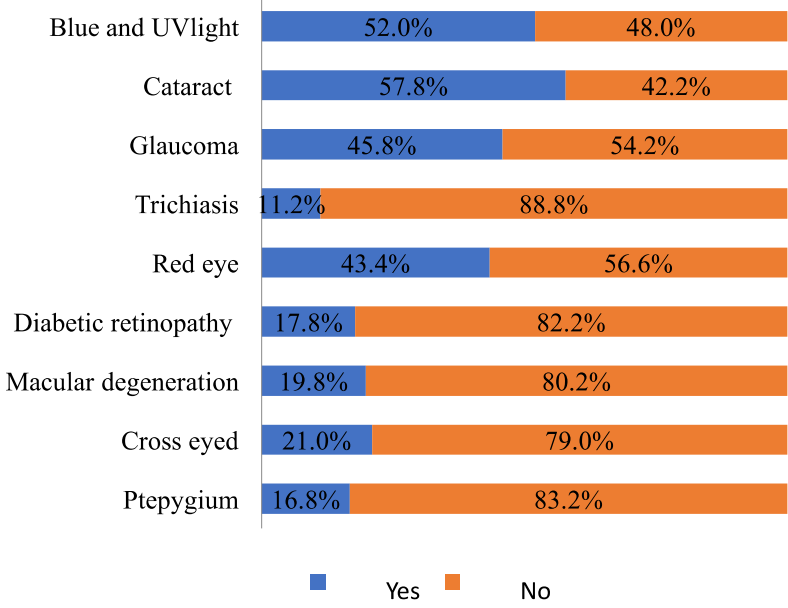

Figure 1: Attendance of some terms by the participants

\section{Comparison between electronic device users and non- users according to gender}

The results revealed that out of 500 participants, computer user was (16\%), smartphone/tablet/iPad user was $(55.6 \%)$, game player $(16 \%)$ and television user (92.8\%). There was only a statistical significant difference at $(P<0.05)$ for game player users (Table 2$)$. On the other hand, the results revealed that the association between male and female participants in related to electronic devices bothering, out of 500 participants; those that are bothering from using computer was (16.2\%), smartphone/tablet/iPad user was (49.2\%), game player (15.4\%) and television user (92\%). There was a non-significant difference between male and female participants (Table 3).

Table 2: Using of electronic devises among participants according to gender.

\begin{tabular}{|c|c|c|c|c|c|}
\hline \multirow{2}{*}{ Electronic devices } & & Male & Female & \multirow{2}{*}{ Pvalue } & \multirow{2}{*}{$\frac{\text { Total }}{\text { No. }(\%)}$} \\
\hline & & No. $(\%)$ & No. $(\%)$ & & \\
\hline \multicolumn{6}{|l|}{ Computer/laptop } \\
\hline & Yes & $48(9.6)$ & $35(7.0)$ & & $83(16.6)$ \\
\hline No & & $247(49.4)$ & $170(34.0)$ & 0.81 & $417(83.4)$ \\
\hline \multicolumn{6}{|c|}{ Smartphone/ Tablet /iPad } \\
\hline & Yes & $163(32.6)$ & $115(23.0)$ & & $278(55.6)$ \\
\hline & No & $132(26.4)$ & $90(18.0)$ & 0.85 & $222(44.4)$ \\
\hline \multicolumn{6}{|l|}{ Game player } \\
\hline & Yes & $56(11.2)$ & $24(4.8)$ & & $80(16.0)$ \\
\hline No & & $239(47.8)$ & $181(36.2)$ & $\leq 0.05$ & $420(84.0)$ \\
\hline \multicolumn{6}{|l|}{ TV } \\
\hline Yes & & $271(54.2)$ & $193(38.6)$ & & $464(92.8)$ \\
\hline No & & $24(4.8)$ & $12(2.4)$ & 0.33 & $36(7.2)$ \\
\hline
\end{tabular}

\begin{tabular}{|c|c|c|}
\hline Characteristics & No. & $\mathbf{( \% )}$ \\
\hline Gender & & \\
Male & 295 & $\mathbf{( 5 9 . 0 )}$ \\
Female & 205 & $(41.0)$ \\
Age (Year) & & \\
$<20$ & 110 & $(22.0)$ \\
$20-39$ & 114 & $(22.8)$ \\
$40-59$ & 125 & $(25.0)$ \\
$60-79$ & 137 & $(27.4)$ \\
$>80$ & 14 & $(2.8)$ \\
Education & & \\
Illiterate & 160 & $(32.0)$ \\
Primary & 145 & $(29.0)$ \\
High school & 128 & $(25.6)$ \\
University & 67 & $(13.4)$ \\
Marital status & & \\
Married & 326 & $(65.2)$ \\
Single & 174 & $(34.8)$ \\
Residence & & \\
Urban & 336 & $(67.2)$ \\
Semi-urban & 55 & $(11.0)$ \\
Rural & 109 & $(21.8)$ \\
Occupation & & \\
Unemployed & 221 & $(44.2)$ \\
Governmental & 68 & $(13.6)$ \\
Employee & 113 & $(22.6)$ \\
Student & 98 & $(16.6)$ \\
Self-employment & & \\
\hline Eye botherng & & \\
\hline Ind & & \\
\hline
\end{tabular}

Table 3: Eye bothering by using of electronic devises among participants according to gender.

\begin{tabular}{|c|c|c|c|c|c|}
\hline \multirow{2}{*}{ Electronic devices } & & Male & Female & \multirow{2}{*}{$P$ value } & \multirow{2}{*}{$\frac{\text { Total }}{\text { No. }(\%)}$} \\
\hline & & No. (\%) & No. $(\%)$ & & \\
\hline \multicolumn{6}{|l|}{ Computer/laptop } \\
\hline & Yes & 44(8.8) & $37(7.4)$ & \multirow[b]{2}{*}{0.34} & $81(16.2)$ \\
\hline & No & $251(50.2)$ & $168(33.6)$ & & $419(83.8)$ \\
\hline \multicolumn{6}{|c|}{ Smartphone /Tablet/iPod } \\
\hline & Yes & $139(27.8)$ & $107(21.4)$ & \multirow{2}{*}{0.26} & $246(49.2)$ \\
\hline & No & $156(31.2)$ & $98(19.6)$ & & $454(50.8)$ \\
\hline \multicolumn{6}{|l|}{ Game player } \\
\hline & Yes & $54(10.8)$ & $23(4.6)$ & \multirow{2}{*}{0.31} & $77(15.4)$ \\
\hline & No & $241(48.2)$ & $182(36.4)$ & & $423(84.6)$ \\
\hline \multicolumn{6}{|l|}{ TV } \\
\hline & Yes & $268(53.6)$ & $192(38.4)$ & \multirow{2}{*}{0.25} & $460(92.0)$ \\
\hline & No & $27(5.4)$ & $13(2.6)$ & & $40(8.0)$ \\
\hline
\end{tabular}

\section{Knowledge and practices of participants with special ophthalmological problems}

Participants with some ophthalmological problems were asked about their knowledge and practices that related with eye diseases. the study explored that the patients with sensitivity to sunlight, dry eye, glaucoma, and eye strain have statistical significant correlation with knowledge about the UV/ blue lights and its risks on eyes. Furthermore, only the patients with sun light sensitivity and cataract had significantly knowledge about the effects of electromagnetic wave of smartphone/tablet /iPad on human eyes. Moreover, the patients with dry eyes, eye/lid infection and glaucoma had significantly more practice for television visual breaks as compared with the other patients. While patients with sun light sensitivity, dry eyes, eye/lid infection and cataract had significantly more practice for taking visual breaks during working on 
smartphone/tablet/iPods. Surprisingly, only patients with dry eyes had visual break taking during computer using (Table 4).

Table 4: Knowledge and practices of participants that associated with ophthalmological problems.

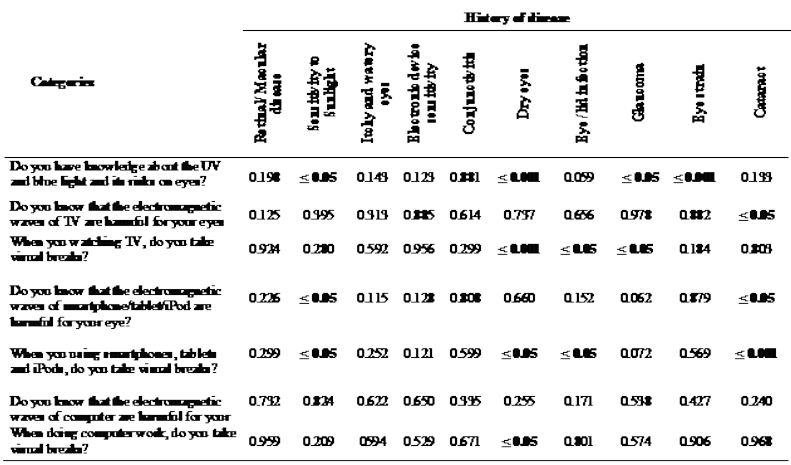

\section{DISCUSSION}

Information about public knowledge and behaviours regarding to eye diseases in general and protection measures among Kurdish people are little and none official study in Kurdistan was found after a broad literature review on this topic. The climate of Kurdistan has distinct high temperatures in the day-time and low temperatures during the night-time. From June to September, daytime temperatures reach $40{ }^{\circ} \mathrm{C}$ or higher throughout the area. The south has higher temperatures, which can reach as high as $48^{\circ} \mathrm{C}$ during summer time. Furthermore, the technological apparatus such as TV, iPad, smartphone, and computer are currently using by the people. There is not more information about the effect of those equipment on human eyes because all of these apparatuses radiate electromagnetic radiation and this radiation has the negative effect on eye [8].

For understanding the knowledge of the participants to some scientific terms (Figure 1 and Table 1), most of the participants had information about blue light, UV light, red eye, cataract, and glaucoma, but there was a significant difference between the male and the females only for blue and UV light knowledge $(\mathrm{P}<0.05)$, this is may be referred to the society education and the study population. There are many studies demonstrated knowledge of people about UV light $[9,10,11]$.

The study findings are potentially valuable to help guide public policy and target interventions. The participants of our study were asked about knowledge and practices related with eye diseases and the study explored that the patients with sensitivity to sunlight, dry eye, glaucoma, and eye strain have statistical significant correlation with

knowledge about the UV/ blue lights and its risks on eyes. Furthermore, only the patients with sun light sensitivity and cataract had significantly knowledge about the effects of electromagnetic wave of smartphone/tablet /iPad on human eyes. Also, the patients with dry eyes, eye/lid infection and glaucoma had significantly more practice for television visual breaks as compared with the other patients. While patients with sun light sensitivity, dry eyes, eye/lid infection and cataract had significantly more practice for taking visual breaks during working on smartphone/tablet/iPods. Surprisingly, only patients with dry eyes had visual break taking during computer using (Table.4).

\section{CONCLUSION}

This research concluded that the patients who visited Shahid Aso eye hospital regarding to eye diseases and radiation, the harmful effects of UV/blue light and sun protection methods showed a low level of public attendance and self-care practice. Additionally, this work has discovered that sun protection measure is commonly inadequate among the current study population and on a regular basis only a small part of participants uses sun protection requirements.

\section{REFERENCE}

[1] DM. Barbu, "The Effects of Radiation on the Eye in Industrial Environments," Annals of the Oradea University. 2015;14(2):7-12

[2] A. Shapiro, "Understanding blue light. Exposure to certain wavelengths of blue light carries potential blinding effects and a link to AMD,” APRIL 2016.

[3] M. Dunbar, R. Melton, "The lowdown on blue light: Good vs bad and its connection to AMD," Review of optometry. www.reviewofoptometry.com/continuing education. November 21, 2013. Accessed March 18, 2016.

[4] J. Gallas, M. Eisner, "Eye protection from sunlight damage. Comprehensive Series in Photosciences,” 3: Elsevier; 2001. p. 437-55.

[5] W. Williams, "Effects of ultraviolet (UV) light on the Eye." IPSWICH01473252595 www.observatory.co.uk.

[6] SC. Saccà, AM. Roszkowska, A. Izzotti, "Environmental light and endogenous antioxidants as the main determinants of noncancer ocular diseases," Mutation Research/Reviews in Mutation Research. 2013;752(2):153-71.

[7] TJ. Berg, "Intraocular light scatter, reflections, fluorescence and absorption: what we see in the slit lamp," Ophthalmic and Physiological Optics.2018;38(1):6-25.

[8] L. Kirk, S. Greenfield, "Knowledge and attitudes of UK university students in relation to ultraviolet radiation (UVR) exposure and their sun-related behaviours: a qualitative study”, BMJ open. 2017;7(3): e014388.doi:10.1136/bmjopen-2016014388 .

[9] E. Bogumiła Zuba, W. Francuzik, P. Malicki, A. OsmolaMańkowska, D. Jenerowicz, "Knowledge about ultraviolet radiation hazards and tanning behavior of cosmetology and medical students," Acta dermatovenerologica Croatica. 2016;24(1):73-77.

[10] RP. Gallagher, TK. Lee, CD. Bajdik, M. Borugian, "Ultraviolet radiation," Chronic Diseases and Injuries in Canada. 2010;29:(51-68).

[11] JA. Choi, K. Han, Y-M. Park, TY. La, "Low serum 25hydroxyvitamin D is associated with myopia in Korean adolescents,"Investigative ophthalmology \& visual science. 2014;55(4):2041-7. 